\title{
A case of individual variation of the rhomboid muscles
}

\author{
M. Ulkir'10, M.F. Sargon² ${ }^{2}$ \\ ${ }^{1}$ Department of Anatomy, Faculty of Medicine, Hacettepe University, Ankara, Turkey \\ 2Department of Anatomy, Faculty of Medicine, Lokman Hekim University, Ankara, Turkey
}

[Received: 9 January 2020; Accepted: 1 February 2020]

\begin{abstract}
During the routine gross anatomic dissection of a Turkish male cadaver; a variation of rhomboid muscles was observed on the left side. There were two rhomboid minors and three rhomboid majors coursing under the trapezius muscle. The origins of the upper and lower rhomboid minor muscles were C5, C6 and C7 vertebrae, respectively. Their insertions were to medial border of scapula, to upper part and to lower part of the spine of scapula, respectively. The origins of the upper, middle and lower rhomboid majors were C7, T1-T3 and T4-T5 vertebrae, respectively. Their insertions were to the 2/3 most inferior part of the medial border of scapula, from superior to inferior in sequence. In the examination of the literature, we could not observe such a variation of these muscles. In surgical procedures these types of variations have a clinical importance for intrathoracic muscle flap transfers and in cases with the paralysis of trapezius muscle. (Folia Morphol 2021; 80, 1: 222-224)
\end{abstract}

Key words: dissection, anatomy, cadaver

\section{INTRODUCTION}

The rhomboid minor and rhomboid major muscles are inferior to levator scapulae muscle. Rhomboid minor is a small, cylindrical muscle and rhomboid major is a quadrilateral sheet of muscle [8]. These two muscles work together to retract or pull the scapula towards the vertebral column. With other muscles; they may also rotate lateral aspect of the scapula inferiorly [1]. In the literature; there are only a few reports related with the variation of these two muscles $[4,5,9]$. In the present case; to our knowledge, we report a unique variation of these two muscles on the left side of a cadaver.

\section{CASE REPORT}

During the routine gross anatomic dissection of the superficial back muscles of a 67-year-old Turkish male cadaver, a variation of rhomboid muscles was observed on the left side. There were two rhomboid minors and three rhomboid majors coursing under the trapezius muscle. The origins of the upper and lower rhomboid minor muscles were C5, C6 and C7 vertebrae, respectively. Their insertions were to medial border of scapula, to upper part and to lower part of the spine of scapula, respectively. The origins of the upper, middle and lower rhomboid majors were C7, T1-T3 and T4-T5 vertebrae, respectively. Their insertions were to the $2 / 3$ most inferior part of the medial border of scapula, from superior to inferior in sequence. In the examination of the literature, we could not observe such a variation of these muscles. In the cadaver, the left superficial back muscles, the right half of the hemithorax and right superficial back muscles were normal in every aspect (Fig. 1). There was any abnormality on the cadaver and it was a healthy specimen. 


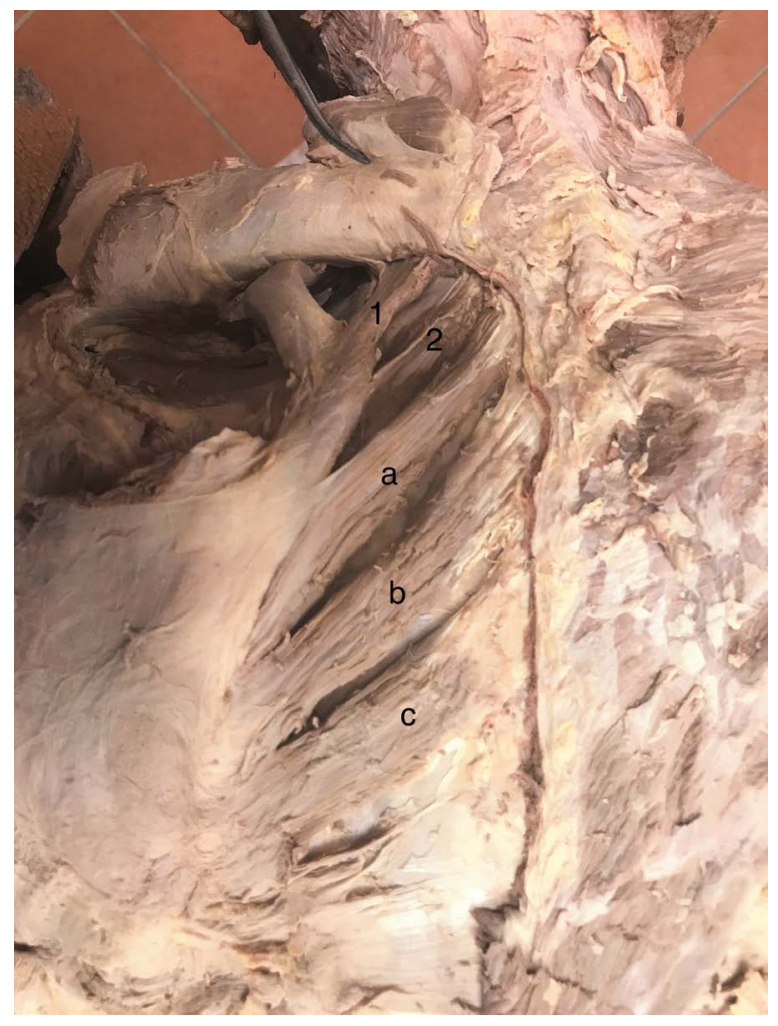

Figure 1. Variation of rhomboid muscles; 1, 2 - rhomboid minor muscles; a, b, c - rhomboid major muscles.

\section{DISCUSSION}

The variations of the rhomboid muscles are very rare. During the superficial back dissection of a 72-year-old male cadaver, Jelev and Landzhov [4] observed an aberrant muscle bilaterally below the lower border of the rhomboideus major. The unusual muscle arose by a thin aponeurosis from the spinous process of the mid-thoracic vertebrae and was attached laterally to the lowest part of the medial border of the scapula [4]. In the study of Mori [5], a muscular slip called "musculus rhomboideus minus" was described in $11-14 \%$ of the Japanese population. This muscle was originated from the spinous process above the latissimus dorsi origin, was coursing laterally and gradually disappearing or passing into the fascia of the teres major muscle [5]. Von Haffner [9] observed a small muscle on the right side of a cadaver, stretched between the sixth thoracic vertebra and the inferior angle of the scapula. The author named this muscle as "musculus rhomboideus minimus". Additionally, in his case, bilateral incomplete agenesis of the trapezius muscles was present [9]. In another study, Saberi et al. [7] investigated the embryonic origins of the rhomboid muscles by following the derivatives of medial and lateral somite halves using quail-chick chimeras. Their results showed that the rhomboid muscles were made up of cells derived mainly from the lateral portion of the somite [7].

In animals: the rhomboid muscles always present cervical and thoracic parts and in carnivores there is an additional, capital part. Rhomboid muscles are innervated from the brachial plexus in the dog, but in some species they are also innervated by dorsal branches of local spinal nerves [2].

In the examination of the literature, our variation is unique and is clinically important. When found during surgical procedures, it can be used together with the usual rhomboid muscles for intrathoracic muscle flap transfers [3]. Additionally, the transfer of the rhomboid muscles to the infraspinous fossa (the Eden-Lange procedure) is important in cases with the paralysis of the trapezius muscle [6].

\section{CONCLUSIONS}

In the literature, variations of rhomboid muscles are scarce. The present case report describes a variation of the rhomboid muscles. In surgical procedures these types of variations have a clinical importance for intrathoracic muscle flap transfers and in cases with the paralysis of trapezius muscle.

\section{Acknowledgements}

The authors of this case report declare no relationships with any companies, whose products or services may be related to the subject matter of the article. No complex statistical methods were necessary for this paper. The cadaver belongs to the Hacettepe University, Faculty of Medicine, Department of Anatomy, Turkey.

\section{REFERENCES}

1. Chang KV, Wu WT, Mezian K, et al. Sonoanatomy of muscles attaching to the medial scapular border (levator scapulae, rhomboid minor, and serratus anterior) revisited. Am J Phys Med Rehabil. 2019; 98(7): e79-e80, doi: 10.1097/PHM.0000000000001136, indexed in Pubmed: 30640725.

2. Dyce KM, Sack WO, Wensing CJG. Textbook of Veterinary Anatomy. 4. ed. Saunders 2009: p. 84.

3. Grima R, Krassas A, Bagan P, et al. Treatment of complicated pulmonary aspergillomas with cavernostomy and muscle flap: interest of concomitant limited thoracoplasty. Eur J Cardiothorac Surg. 2009; 36(5): 910-913, doi: 10.1016/j. ejcts.2009.05.007, indexed in Pubmed: 19595606.

4. Jelev L, Landzhov B. A rare muscular variation: the third of the rhomboids. Anatomy. 2013; 6-7: 63-64, doi: 10.2399/ ana.11.218.

5. Mori M. Statistics on the musculature of the Japanese. Okajimas Folia Anat Jpn. 1964; 40: 195-300, doi: 10.2535/ ofaj1936.40.3_195, indexed in Pubmed: 14213705. 
6. Romero J, Gerber C. Levator scapulae and rhomboid transfer for paralysis of trapezius. The Eden-Lange procedure. J Bone Joint Surg Br. 2003; 85(8): 1141-1145, doi: 10.1302/0301-620x.85b8.14179, indexed in Pubmed: 14653596.

7. Saberi M, Pu Q, Valasek P, et al. The hypaxial origin of the epaxially located rhomboid muscles. Ann Anat. 2017; 214: 15-20, doi: 10.1016/j.aanat.2017.05.009, indexed in Pubmed: 28655569.
8. Standring S. Gray's Anatomy. 41. ed. Elsevier 2016: p. 818.

9. Von Haffner H. Eine seltene doppelseitige Anomalie des Trapezius. Internationale Monatsschrift für Anatomie und Physiologie. 1903; 20: 213-218 (as cited by Jelev L, Landzhov B. A rare muscular variation: the third of the rhomboids. Anatomy 2013; 6-7: 63-64, doi: 10.2399/ ana.11.218). 